\title{
Bayesian Semi-Parametric Regression Analysis of Childhood Malnutrition in Gamo Gofa Zone: The Social and Economic Impact of Child Undernutrition
}

\author{
Tilahun Ferede Asena ${ }^{*}$, Derbachew Asfaw Teni \\ Department of Statistics, Arba Minch University, Arba Minch, Ethiopia \\ Email address: \\ feredetilahun14@gmail.com (T. F. Asena),dasfaw469@gmail.com (D. A. Teni) \\ To cite this article: \\ Tilahun Ferede Asena, Derbachew Asfaw Teni. Bayesian Semi-Parametric Regression Analysis of Childhood Malnutrition in Gamo Gofa \\ Zone: The Social and Economic Impact of Child Undernutrition. American Journal of Theoretical and Applied Statistics. \\ Vol. 4, No. 4, 2015, pp. 269-276. doi: 10.11648/j.ajtas.20150404.17
}

\begin{abstract}
Major progress has been made over the last decades in reducing the prevalence of malnutrition amongst children less than 5 years of age in developing countries. Approximately $27 \%$ of children under the age of 5 in these countries are still malnourished. This work focuses on the childhood malnutrition in Gamo Gofa Zone, Ethiopia. This study examined the association between demographic and socioeconomic determinants and the malnutrition problem in children less than 5 years of age using Data obtained from both rural and urban sampled surveys conducted in sample Woredas from December 1 to January 5, 2013. The study on the Child undernutrition and underweight prevalence in Gamo Gofa has allowed us to quantify the negative impacts of child undernutrition in both social and economic terms. The results revealed that as many as $75 \%$ of all cases of child undernutrition and its related pathologies go untreated. It is also observed that about $35 \%$ of the health costs associated with undernutrition occur before the child turns 1 year-old. Generally, The results of the analysis show that place of residence, employment status of mother, employment status of partners, educational status of mothers, diarrhea, household economic level and source of drinking water were found to be the most important determinants of health/nutritional status of children. The study revealed that socio-economic, demographic and health and environmental variables have significant effects on the nutritional and health status of children in Ethiopia.
\end{abstract}

Keywords: Bayesian Models, Childhood Malnutrition, Ethiopia, Gamo Gofa Zone

\section{Introduction}

The poor nutritional status of children and women has been a serious problem in Ethiopia for many years. Numerous socioeconomic and cultural factors influence patterns of feeding children and the nutritional status of women and children. The period from birth to age two is especially important for optimal growth, health, and development. Unfortunately, this period is often marked by micronutrient deficiencies that interfere with optimal growth. Additionally, childhood illnesses such as diarrhea and acute respiratory infections (ARI) are common.

Nutrition and health are important dimensions of human well-being. Malnutrition represents the main health problem in developing countries. Various studies in different/same countries may find different results over the importance of the determinant factors behind children's nutrition and health outcomes. Estimates may differ depending on various factors including the nature of the data and estimating methodology. The extent of child malnutrition and prevalence varies within societies and also among different clusters and areas of people. The factors responsible for the variation are at different hierarchy (individual, family (cluster), and community (regional) levels), embedded in socio-economic, demographic and cultural Society of Ethiopia. Therefore, this study has been motivated to address the identified research gaps by answering the following

- What are the key factors that affect Children's Nutritional status at each level within each woredas?

- What are the key factors that influence Childhood Undernutrition and Underweight Prevalence in Gamo Gofa Zone?

- How important is this factor in relation to other socioeconomic, demographic and cultural factors?

- Is there any rural urban difference in Childhood Undernutrition and Underweight Prevalence in Gamo 


\section{Gofa Zone?}

- How much variations are measured in Childhood Undernutrition and Underweight

The main purpose of this study is to determine key factors affecting childhood Undernutrition and Underweight Prevalence in Ethiopia, Specifically in Gamo Gofa zone. The Specific objectives are:-

- To investigate how socio-economic, demographic, health and environmental characteristics affect childhood under-nutrition and underweight.

- To assess the impact of under nutrition and underweight within urban and rural areas.

- To assess the variation in relation to factors influencing childhood under-nutrition and Underweight prevalence.

- To provide relevant recommendations for policy makers and suggest directions for future studies.

\section{Methods and Materials}

Data obtained from both rural and urban sampled surveys conducted in Gamo Gofa zones of Dita, Boreda, Dembi Gofa and Mirab Abaya Woredas from December 1 to January 5, 2013. The data is collected from four woredas among which 12 kebeles are selected from these woredas.In this study a total of 564 children under age five (6-59 months) are considered for the analysis. Because of the study focusing on household surveys, the sample size is calculated in terms of the number of children under the age five that should be selected. The summary of sample data is displayed in Table 1 where, $M_{i}=$ Number of children under the age five within $i^{\text {th }}$ kebeles, pi stands for Probability of selecting of $\mathrm{j}^{\text {th }}$ child under the age five in $i^{\text {th }}$ kebeles and $n_{i}$ is the sample size calculated in $\mathrm{i}^{\text {th }}$ kebele.

Table 1. Number of Children under the Age Five sampled from Each PSU of Childhood Malnutrition in Gamo Gofa Zone (2013).

\begin{tabular}{llll}
\hline Name of cluster(Woredas) & $\mathbf{M}_{\mathbf{i}}$ & $\mathbf{P}_{\mathbf{i}}$ & $\mathbf{n}_{\mathbf{i}}$ \\
\hline Dita & 1229 & 0.0632 & 155 \\
Boreda & 726 & 0.0373 & 130 \\
Denba Gofa & 763 & 0.0392 & 158 \\
Mirab Ababay & 858 & 0.0441 & 121 \\
Total & - & - & 564 \\
\hline
\end{tabular}

Response variables: Three continuous response variables are considered for analysis of childhood malnutrition and underweight prevalence. These are:-Stunting: (Height-for-age, which indicates stunting), Underweight: (Weight-for-age, an indication of underweight) and Wasting: (Weight-for-height, an indication of wasting).The following formula for Z-score was used to carry out the analysis of children's malnutrition. This is represented as: $Z_{i j}=\frac{X_{i j}-\mu_{j}}{\sigma_{j}}$ Where: $\mathrm{i}=$ reference to individuals (Children), $\mathrm{j}=1, \ldots, 3$ with $Z_{1}=\mathrm{Z}$-Score height- forage nutrition index, $Z_{2}=\mathrm{z}$-Score weight- for-age nutrition index and $Z_{3}=\mathrm{z}$-Score height- for- weight nutrition. $X_{i j}$ is observed value of the $i^{\text {th }}$ child. $\mu_{j}$ is the mean value of the reference population and $\sigma_{j}$ is the standard devotion of reference population.

Continuous Covariates: - Covariates which are assumed to be determinant factors for childhood malnutrition and underweight prevalence are displayed as follows

Table 2. Some Explanatory Variables with their Codes of Childhood Malnutrition in Gamo Gofa Zone (2013).

\begin{tabular}{ll}
\hline Factor & Code \\
\hline Woredas & Woreda \\
Type of place of residence & Residance \\
Highest educational level & Educ \\
Source of drinking water & D_Water \\
Type of toilet facility & T_ToiFacility \\
Number of children 5 and under in household (de jure) & No_Child \\
Sex of household head & Sex_HH \\
Age of household head & Age_HH \\
Wealth index & Wealth_index \\
Total children ever born & Tot_Child \\
Births in last five years & Birth_5yrs \\
Age of Mothers at 1st birth & M_Age \\
Number of living children & Child_Liv \\
Mothers weight in kilograms $(1$ decimal) & Moth_Wieght \\
Mothers height in centimeters $(1$ decimal) & Moth_Hieght \\
Body Mass Index & BMI \\
Women's age in years (from household questionnaire) & Wom_Age \\
Current marital status & Marital_Stat \\
Husband/partner's education level & Part_Educ \\
Respondent currently working & Cur_Work \\
Husband/partner's occupation & Part_Ocup \\
Birth order number & Birth_order \\
Child is twin & Child_Twin \\
Sex of child & Sex_child \\
Duration of breastfeeding & Breastfed_Dur \\
Months of breastfeeding & Month_Breasfed \\
Child's age in months & C_age \\
Child's weight in kilograms $(1$ decimal) & Child_wieght \\
Child's height in centimeters $(1$ decimal) & Child_hieght \\
\hline & \\
& \\
\hline &
\end{tabular}

Bayesian Geo-Additive Regression Models: Consider regression situations, where observations $\left(Y_{i}, X_{i}, W_{i}\right)$ $i=1,2, \ldots, n$ on a metrical response $\mathrm{Y}, \quad$ a vector $X=\left(x_{1}, x_{2}, \ldots ., x_{p}\right)$ of continuous covariate and a vector $W=\left(w_{1}, w_{2}, \ldots ., w_{r}\right)$ of discrete covariates are given then effect of the covariates on the response is modelled by a linear predictor given by:

$$
\eta_{i}=X_{i}^{T} \beta+W_{i}^{T} \gamma
$$

Spatial analyses of undernutrition often are confined to using region-specific (areal) dummy variables to capture the spatial dimension. Here, we went a step further by exploring regional patterns of childhood undernutrition and, possibly nonlinear, effects of other factors within a simultaneous, coherent regression framework using a geo-additive semi parametric mixed model. Because the predictor contains usual linear terms, nonlinear effects of metrical covariates and geographic effects in additive form, such models are also called geo-additive models. Kammann and Wand (2003) proposed this type of models within an empirical Bayesian approach. Here, we apply a fully Bayesian approach as 
suggested in Fahrmeir and Lang (2001), Lang and Brezger (2004) which is based on Markov priors and uses MCMC techniques for inference and model checking. Thus, we

$$
\eta_{i}=f_{2}\left(X_{i 1}\right)+f_{2}\left(X_{i 2}\right)+, \ldots \ldots . .,+f_{p}\left(X_{i p}\right)+f_{s p a t}\left(s_{i}\right)+\beta+W_{i}^{T} \gamma
$$

Here $f_{1}, \ldots . ., f_{p}$ are nonlinear smooth effects of the metrical covariates and $f_{\text {spat }}$ is the effect of district $s_{i} \in(1 \ldots \ldots, s)$ where mother ilives. In a further step we may split up the spatial effect $f_{\text {spat }}$ into a spatially correlated (structured) and an uncorrelated (unstructured) effect

$$
f_{\text {spat }}\left(s_{i}\right)=f_{\text {stra }}\left(s_{i}\right)+f_{\text {unstru }}\left(s_{i}\right)
$$

If the unstructured effect exceeds the structured effect, the spatial dependency is smaller and vice versa. Such models are common in spatial epidemiology; see e.g. Besag et al. (1991).

Bayesian Semi-Parametric Regression Analysis: The assumption of a parametric linear predictor for assessing the influence of covariate effects on responses seems to be rigid and restrictive in practical application situation and also in many real statistically complex situation since their forms cannot be predetermined a priori. In this application to childhood under-nutrition and in many other regression replace the strictly linear predictor (1) by the more flexible semiparametric predictor

situations, we are facing the problem for the continuous covariates in the data set; the assumption of a strictly linear effect on the response Y may not be appropriate as suggested in Fahrmeir and Lang (2001)and Fahrmeir and Lang (2004). Traditionally, the effect of the covariates on the response is modelled by a linear predictor as:

$$
\eta_{i}=X_{i}^{T} \beta+W_{i}^{T} \gamma_{i}
$$

Where, observation $\left(Y_{i} X_{i}, W_{i}\right) \quad i=1,2, \ldots, n$ on a metrical response $\mathrm{Y}$, a vector $X=\left(x_{1}, x_{2}, \ldots, x_{p}\right)$ of metrical covariate, $\beta=\left(\beta_{1}, \beta_{2}, \ldots ., \beta_{p}\right)$ is a vector of regression coefficients for the continuous covariates. $\gamma=\left(\gamma_{1}, \gamma_{2}, \ldots, \gamma_{r}\right)$ is a vector of regression coefficients for the categorical covariates and a vector $W=\left(w_{1}, w_{2}, \ldots ., w_{r}\right)$ of categorical covariates. The semi-parametric regression model is obtained by extending model (5) as follows:

$$
\eta_{i}=f_{2}\left(X_{i 1}\right)+f_{2}\left(X_{i 2}\right)+, \ldots \ldots \ldots,+f_{p}\left(X_{i p}\right)+f_{s p a t}\left(s_{i}\right)+\beta+W_{i}^{T} \gamma
$$

Where $: f_{1}, \ldots ., f_{p}$ are smooth effects of the continuous covariates.

Prior Distributions: In model (6), the parameters of interest $f_{j}, j=1, \ldots \ldots, P$ and $\gamma_{i}$ parameters as well as the variance parameter $\left(\tau^{2}\right)$ are considered as random variables and have to be supplemented with appropriate prior assumptions. Suppose that $f=\left(f\left(X_{1}\right), f\left(X_{2}\right), \ldots, f\left(X_{n}\right)\right)^{\prime}$ is the vector of corresponding function evaluations at observed values of $\mathrm{X}$. Then, the prior for $\mathrm{f}$ is

$$
\left[f \mid \tau^{2}\right] \propto \exp \left(-\frac{1}{2 \tau^{2}} f^{\prime} K f\right)
$$

Where, $\mathrm{K}$ is a penalty matrix that penalizes too abrupt jumps between neighboring parameters. In most cases, $\mathrm{K}$ will be rank deficient; therefore the prior for $\mathrm{f}$ would be improper. This implies that $\left(f \mid \tau^{2}\right)$ follows a partially improper Gaussian prior where $f \mid \tau^{2} \sim N\left(0, \tau^{2} K^{-}\right)$where $K^{-}$is a generalized inverse of a band-diagonal precision or penalty matrix K. it is possible to express the vector of function evaluations $f=\left(f\left(X_{1}\right), f\left(X_{2}\right), \ldots, f\left(X_{n}\right)\right)^{\prime}$ of a nonlinear effect as the matrix product of a design matrix $X_{j}$ and a vector of regression coefficients $\beta_{j}, f_{j}=X_{j}^{\prime} \beta_{j}$ Brezger and Lang (2006) also suggest a general structure of the priors for $\beta_{j}$ as:

$$
P\left(\beta_{J} \mid \tau^{2}\right) \propto \frac{1}{\left(\tau^{2}\right) \operatorname{rank}\left(k_{j}\right) / 2} \exp \left(-\frac{1}{2 \tau^{2}} \beta_{j}^{\prime} K_{j} \beta_{j}\right)
$$

Where, $K_{j}$ is a penalty matrix that shrinks parameters towards zero or penalizes too abrupt jumps between neighboring parameter.Therefore, hyperpriors are assigned to the variances $\tau^{2}$ in a further stage of the hierarchy by highly dispersed (but proper) inverse Gamma priors $P\left(\tau^{2}\right) \sim$ $I G(a, b)$.

$$
P\left(\tau^{2}\right)=\left(\tau^{2}\right)^{-a-1} \exp \left(-\frac{b}{\tau^{2}}\right)
$$

A common choices for the hyperparameters are small values, for example are $a=1$ and $b=0.005$ (or $b=0.0005$ ). Alternatively, one may take $\mathrm{a}=\mathrm{b}=0.001$ as of Khaled (2010).

Priors for Fixed Effects: In the absence of any prior knowledge for the parameter vector $\gamma$ of fixed effects the study considered a diffuse prior $\gamma \propto$ cons $\tan t j=1, \ldots \ldots, r$. Another choice would be to work with a multivariate Gaussian distribution $\gamma \sim N\left(\gamma_{0}, \sum\right)$. In this study, diffuse priors were used for the fixed effects parameter $\gamma$.

Posterior Probability Distribution: Bayesian inference is based on the entire posterior distribution derived by multiplying the prior distribution $\pi(\theta)$ of all parameters and 
the full likelihood function $L(Y \mid \theta)$. For this case, let $\theta$ be the vector of all unknown parameters, then the posterior distribution is given by:

$$
\begin{gathered}
\pi(\theta \mid Y) \propto L\left(y \mid \beta_{1}, \tau_{1}, \ldots \ldots, \beta_{p}, \tau_{P}, \gamma\right) \prod_{j=1}^{p} \pi\left(\beta_{j} \mid \tau^{2}\right)\left(\tau_{j}^{2}\right) \\
\pi(\theta \mid Y) \propto L\left(y \mid \beta_{1}, \tau_{1}, \ldots \ldots, \beta_{p}, \tau_{P}, \gamma\right)
\end{gathered}
$$

$$
\prod_{j=1}^{p} \frac{1}{\left(\tau^{2}\right) \operatorname{rank}\left(K_{j}\right) / 2} \exp \left(-\frac{1}{2 \tau^{2}} \beta_{j}^{\prime} K_{j} \beta_{j}\right) \prod_{j=1}^{p}\left(\tau^{2}\right)^{-a j=1} \exp \left(-\frac{\beta_{j}}{\tau_{j}^{2}}\right)
$$

In many practical situations (as is the case here) the posterior distribution is numerically intractable. To overcome this problem, Markov Chain Monte Carlo (MCMC) simulation technique is used to draw samples from the posterior. From these samples, quantities such as (posterior) mean, (posterior) standard deviation, and quantiles (which in turn, gives the associated credible interval) can be estimated. In fully Bayesian inference, the unknown variance parameters $\tau^{2}$ are considered as random and estimated simultaneously with the unknown regression parameters. Therefore, hyperpriors are assigned to the variances $\tau^{2}$ in a further stage of the hierarchy by highly dispersed inverse Gamma priors $p\left(\tau^{2}\right) \sim I G(a, b)$.

\section{Results and Discussions}

This study examined the association between demographic and socioeconomic determinants and the malnutrition problem in children less than 5 years of age using Data obtained from both rural and urban sampled surveys conducted in Gamo Gofa zones of Dita, Boreda, Dembi Gofa and Mirab Abaya Woredas from December 1 to January 5, 2013. The data is collected from four woredas among which 12 kebeles are selected from these woredas.In this study a total of 564 children under age five (6-59 months) are considered for the analysis. Among these about 158(28.3 percent) of children are underweight of which about 72(46 percent) are under sever nutritional status. This shows that the prevalence of underweight prevalence in Ethiopia is still in high rate in Ethiopia. Table 2: shows the Distribution of Socioeconomic, Demographic, Health and Environmental related Characteristics versus Underweight in Gamo Gofa Zone in 2013. Based on the results Underweight prevalence in rural areas is about $154(28.23$ percent $)$ and in urban areas of the zone is about 10 (25.14 percent), this descriptive result further shows that there is indeed a significant difference among rural and urban residence of the zone. It is also observed that there is also a dependency of child's nutritional status on the parent's educational status; accordingly parents who attended at least primary education have a better off than those who don't attained education at all. It is shown in the table that cough diarrhea and are the most chronic problems for child undernutrition in Gamo Gofa zone. When compared with their source of drinking water, children who utilize unprotected water for drinking are highly exposed to undernutrition than those who utilize protected one; accordingly among 187 children who are under nutritional status more than a half (about 86 children) are utilizing unprotected drinking water.

Table 3. Distribution of Socioeconomic, Demographic, Health and Environmental related Characteristics versus Underweight in Gamo Gofa Zone in 2013.

\begin{tabular}{llll}
\hline \multirow{2}{*}{ factor } & & \multicolumn{2}{l}{ Underweight Status } \\
\cline { 3 - 4 } Residence & Underweight & Not Underweight \\
& Urban & $154(28.23)$ & $373(70.77)$ \\
& Over all & $10(25.14)$ & $27(74.86)$ \\
& No Education & $102(29.61)$ & $397(69.39)$ \\
Highest & primary & $57(35.84)$ & $254(71.35)$ \\
educational level & Secondary & $0(0.00)$ & $732(64.16))$ \\
& higher & $5(55.55)$ & $4(44.45)$ \\
& Over All & $167(29.61)$ & $397(70.39)$ \\
Source of & Protected & $81(28.53)$ & $203(71.47)$ \\
drinking water & unprotected & $86(31.55)$ & $194(68.45)$ \\
Respondent & Total & $284(50.35)$ & $280(49.65)$ \\
currently & Yes & $53(27.32)$ & $141(72.68)$ \\
working & No & $114(30.81)$ & $256(69.19)$ \\
Sex of child & Male & $90(31.25)$ & $198(68.75)$ \\
& Female & $77(27.90)$ & $199(72.10)$ \\
Birth Order & $1-3$ & $78(36.28)$ & $137(63.72)$ \\
Had cough & More than 3 & $112(32.10)$ & $237(67.90)$ \\
recently & No & $81(28.82)$ & $200(71.18)$ \\
Had diarrhea & Yes & $86(30.39)$ & $197(69.61)$ \\
recently & No & $73(27.45)$ & $193(72.55)$ \\
Had fever in last & Yes & $94(31.55)$ & $204(68.45)$ \\
two weeks & No & $87(32.11)$ & $184(67.89)$ \\
\hline & Yes & $80(27.31)$ & $213(72.69)$ \\
\hline
\end{tabular}

Note: Values in parenthesis indicates the percentage values

Results for Linear fixed effect covariates: The analysis of posterior distribution of the model parameters is analytically intractable, which makes it almost impossible for direct inference. Markov Chain Monte Carlo (MCMC) is therefore used to generate samples from the prior distribution which allow estimation and inference for all parameters to be made. Detail information about this modelling approach for models with geoaddative predictors has been implemented in BayesX, software for Bayesian analysis using MCMC and Restricted Maximum Likelihood technique. Table 3 gives results for the fixed effects (categorical covariates) on the nutritional status of children under age five in Gamo Gofa. The output gives posterior means, posterior variance along with their $95 \%$ credible intervals. Based on the results depicted on Table 4 educational levels, birth order of child, toilet Facility, place of residence, cough, respondent's current work status, diarrhea status of child and fever status of child were found statistically significant at 5\% significance level. But, Sex of child and were found statistically insignificant. 
Table 4. Posterior Estimates of the fixed-effect parameters for Underweight in Gamo Gofa Zone in 2013.

\begin{tabular}{|c|c|c|c|}
\hline Factor & PostMean & PostVar & 95\%Credible Interval \\
\hline $\begin{array}{l}\text { Const } \\
\text { child residence }\end{array}$ & $0.423 *$ & 0.002 & {$[0.25,0.476]$} \\
\hline Rural & $-0.05 * *$ & 7.45 & {$[-1.80,-3.72]$} \\
\hline Urban(ref) & - & - & - \\
\hline \multicolumn{4}{|c|}{ Highest educational level } \\
\hline primary & $0.06^{* *}$ & 1.27 & {$[0.01,0.90]$} \\
\hline Secondary & $0.34 *$ & 5.93 & {$[0.15,3.01]$} \\
\hline higher & $0.35^{*}$ & 3.45 & {$[0.30,2.91]$} \\
\hline No Educ(ref) & - & - & - \\
\hline Protected(ref) & - & - & - \\
\hline unprotected & $0.003 * *$ & 0.4 & {$[0.01,0.5]$} \\
\hline $\begin{array}{l}\text { Is there a toilet } \\
\text { No }\end{array}$ & $\begin{array}{l}\text { icility } \\
0.45^{*}\end{array}$ & 1.05 & {$[0.2,2.5]$} \\
\hline Yes(ref) & - & - & - \\
\hline \multicolumn{4}{|l|}{ Sex of child } \\
\hline Male & 0.75 & 6.3 & {$[1.75,10.0]$} \\
\hline Female(ref) & - & - & - \\
\hline \multicolumn{4}{|l|}{ Birth Order } \\
\hline More than 3 & $0.03 * *$ & 0.55 & {$[0.1, \quad 3.5]$} \\
\hline $1-3(\mathrm{ref})$ & - & - & - \\
\hline \multicolumn{4}{|c|}{ Had cough recently } \\
\hline Yes & $-0.03 * *$ & 0.12 & {$[-6.00, \quad 5.5]$} \\
\hline No(ref) & - & - & - \\
\hline \multicolumn{4}{|c|}{ Had diarrhea recently } \\
\hline Yes & $-0.6 * *$ & 1.75 & {$[-2.0,3.04]$} \\
\hline No(ref) & - & - & - \\
\hline \multicolumn{4}{|c|}{ Had fever in last two weeks } \\
\hline Yes & $0.9 * *$ & 0.05 & {$[0.01, \quad 1.5]$} \\
\hline No(ref) & - & - & - \\
\hline
\end{tabular}

$(* *)$ significance at $0.025 \%,(*)$ significance at 0.05 level of significance

According to the results on Table 4 we observed that parent's educational status is found to be a significant factor for children nutritional status, implying that the chance to be underweight for a children whose age is under five is found to decrease with the increasing the educational level of child's mother. As shown in the results having an educated mother at least primary education contributes to have better nourishment for children under age five. The findings of this study indicate that children whose mothers educational status is illiterate don't attained primary education were more likely to be malnourished as compared to mothers with secondary education and higher educations .in comparison with the sex of children the results of the analysis shows that female children are better nourished than male children. On the other hand, one can observe that underweight is higher for children whose mothers birth order is greater than three is larger than those who have less than three children's in her life span. . The analysis showed that children born after a long birth interval were better off than other children. This may be due to the fact that the parents can take better care of fewer numbers of children and could provide adequate breast milk due to recovery of nutritional status between births. Working status of mothers which mightincrease economic status of household had significant effect on child nutritional status. In this study it is also observed that children Health factors are strong indicators of children's underweight status. Children who experience fever two weeks prior to the survey have high risk to their nutritional status than those who don't have fever in the last two weeks before the survey.

Non-linear effect under a Geoadditive Semi-parametric regression Analysis: Nonlinear effects represented by smoothed functions, are commonly interpreted graphically. Figure 5 shows the smooth function of the children age versus weight-for-age $z$-score. The posterior means together with $75 \%$ and $90 \%$ point wise credible intervals are shown. One can observe that the influence of a child's age on its nutritional status is considerably high in the age range between the ages of 0-27 months with decreasing trend; and then stabilizes.

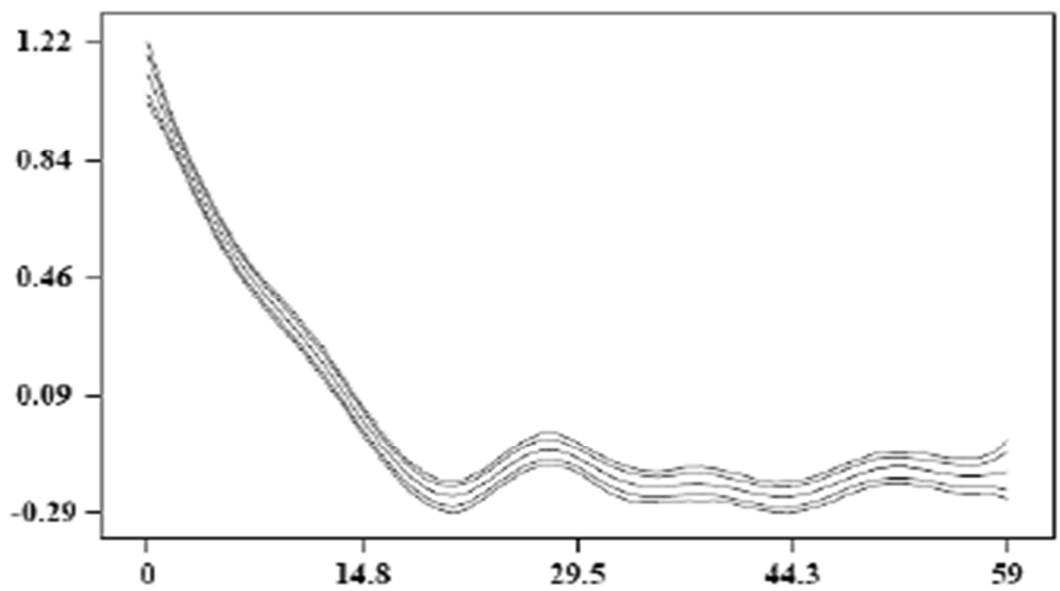

Figure 1. Posterior means of the non-linear effects in underweight for child's age in month Gamo Gofa Zone in 2013.

As suggested by the nutritional literature, one can able to distinguish the continuous worsening of the nutritional status up until about 27 months of age. This deterioration set in right after birth and continues, more or less linearly, until 27 months. After 27 months the effect of age on underweight stabilizes at a low level. Through reduced growth and the waning impact of infections, children were apparently able to reach a low-level equilibrium that allows their nutritional 
status to stabilize.

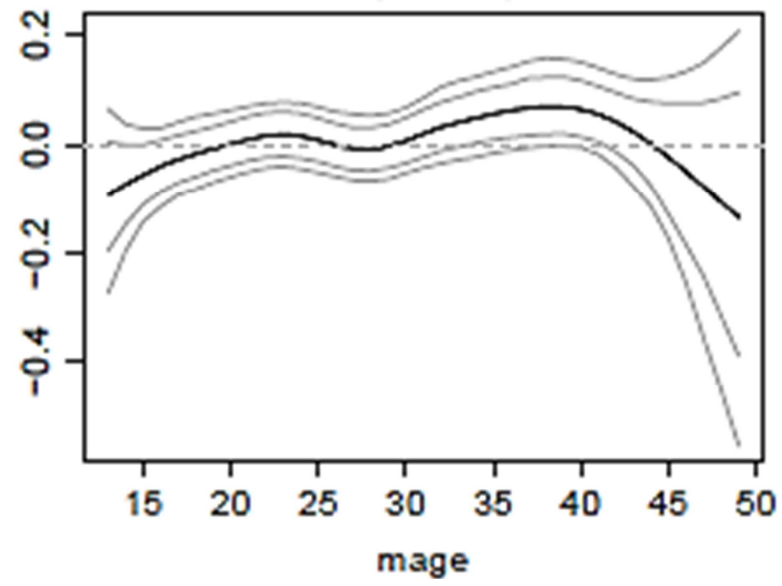

Figure 2. Posterior means of the non-linear effects in underweight for mothers' age at first birth in year's in Gamo Gofa Zone in 2013.

Figure 2 displays nonlinear effects of mother's age at birth in years. It shows the posterior means together with $80 \%$ and
95\% point-wise credible intervals. It is evident from the analysis that increasing age of mother at birth reduces underweight status of children. That is younger mothers tend to have more underweighted children than older mothers. Mother age at birth shows significant effect on underweight status of children under age of five years old. The effect of mother's age on her child's underweight status (other constant) negatively increase as her age increase up to 25 years and then after the effect of mothers age on her child's underweight status positively increase.

Figure 3 shows the flexible modelling of the effect of the BMI kg/m2 of the mother versus underweight. The posterior means together with $80 \%$ and $95 \%$ point wise credible intervals are displayed. Bearing in mind at the mother's BMI $\mathrm{kg} / \mathrm{m} 2$ and its impact on the level of nutritional status, it was found that the influence had a regular pattern. The underweight status of a child improves as mothers BMI increases. In general, the figure shows that BMI kg/m2 had a significant effect on child nutritional status.

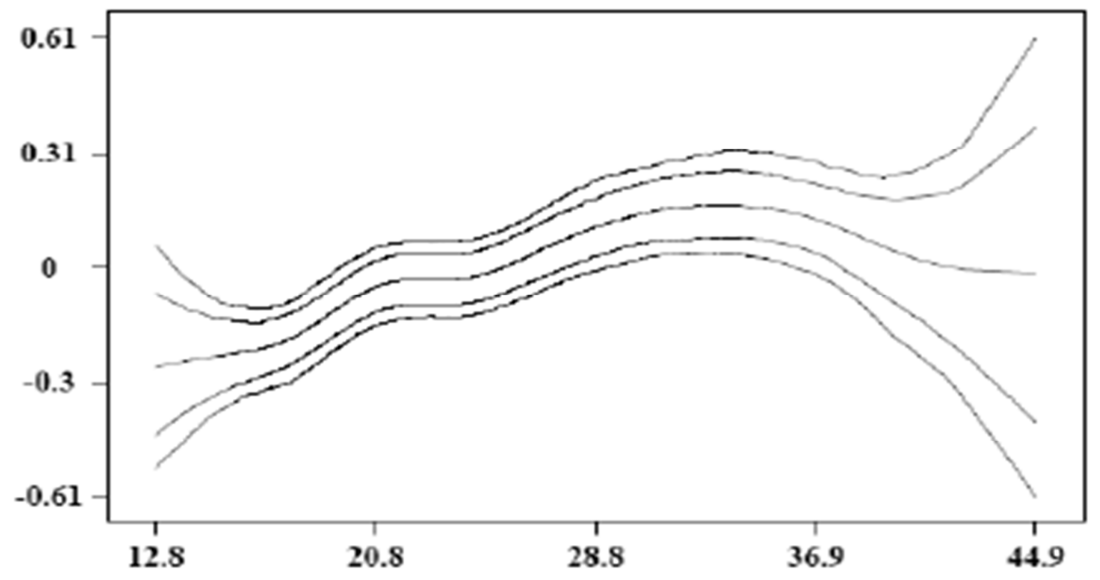

Figure 3. Posterior means of the non-linear effects in underweight for mothers'BMI in Gamo Gofa Zone in 2013.

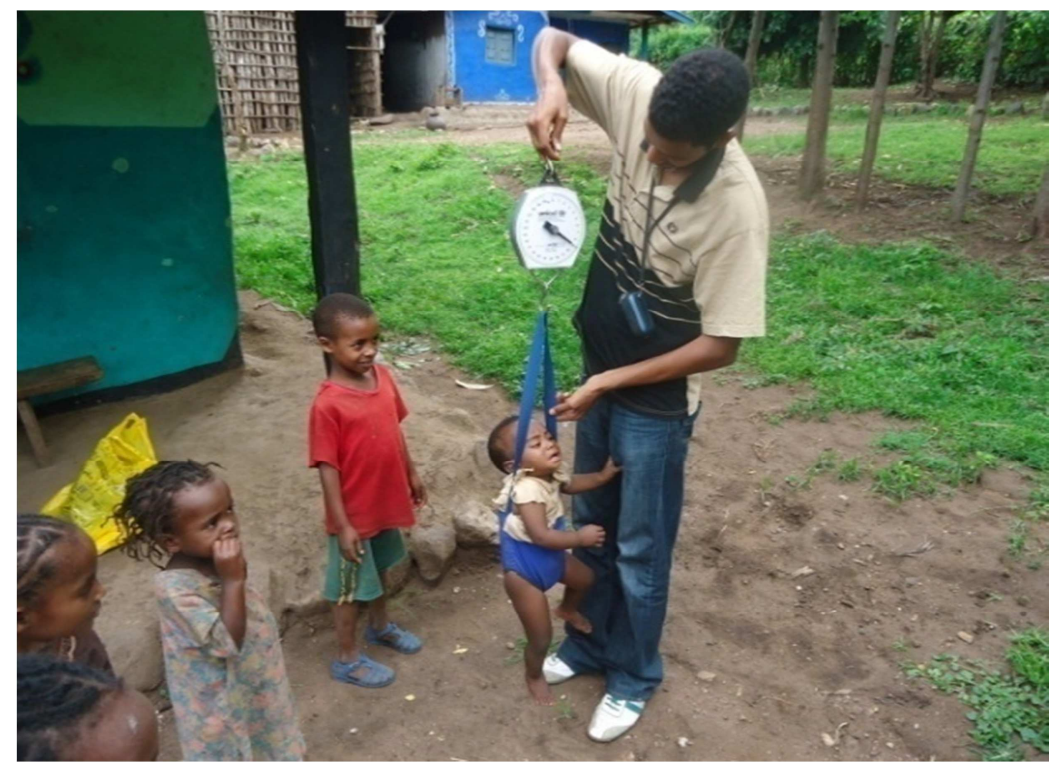

Figure 4(a). While Measuring Child Weight. 


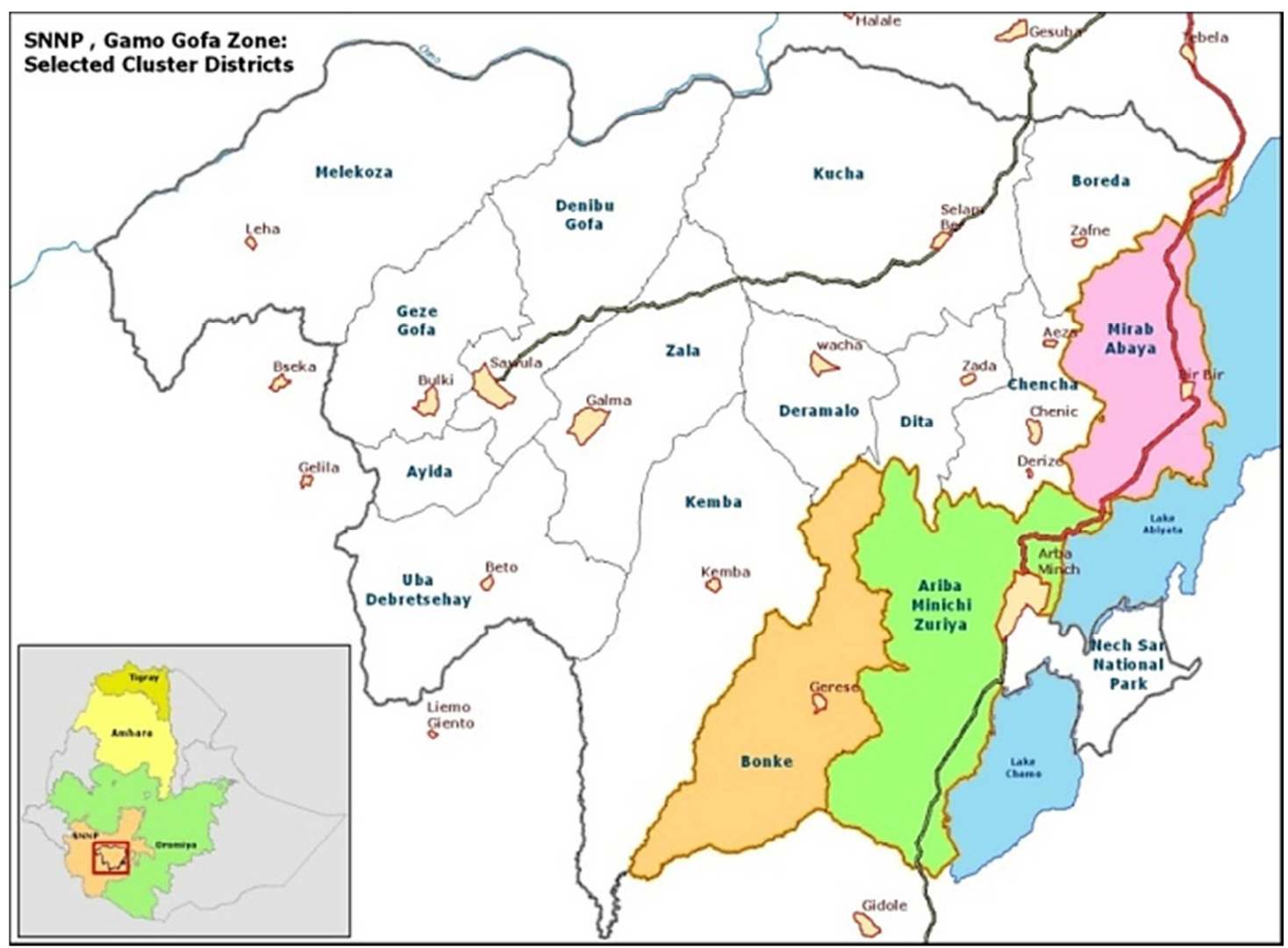

Figure 4(b). The Geographical Map of Gamo Gofa.

Figure 4: shows the geographic map of the typical studies is done. In figure 4(a) the data investigators are measuring the weight of children in the study area and recording the results in provided spread sheet data record.

\section{Concluding Remarks and Future Research}

This study revealed that socio-economic, demographic and proximate variables have important effect on Childhood undernutrition and underweight prevalence in Gamo Gofa zone. Place of Residence, Highest educational level, Source of drinking water, Respondent currently working, Sex of child, Birth Order, Had cough recently, Had diarrhea recently, Had fever in last two weeks were the most important determinants for Childhood undernutrition and underweight prevalence in Gamo Gofa zone. Increasing health care centers and decreasing Childhood undernutrition and underweight prevalence in Gamo Gofa zone in rural areas will directly address challenges of child growth disparities between areas and in rural parts of the Gamo Gofa zone. There is a marked difference between respondent's level of educational and underweight prevalence in Gamo Gofa zone, thus efforts should be made to motivate women to attain school educations to minimize the difference. Although, there is higher rate of underweight prevalence in Gamo Gofa zone it is observed that there is increasing work by the government to achieve the MDG's goal nationally in the year 2015. In the last 10 years the prevalence rate has been decreased steeply.
Women who have education at least primary, secondary and higher school attainments have comparatively significantly good Childhood nutrition than those women who do not have school education at all. This may be because of the fact that educated women are well informed about Childhood undernutrition effects on child growth. In addition, educated ones know the benefit of limiting child nutrition on social and economic impacts with no school educations. Thus, efforts should be made to motivate women to attain school educations to minimize the problems.

Recognizing the significant difference on residence and districts the effect of malnutrition and high prevalence of underweight in the study communities, there is a clear need for carefully designed longitudinal study to definitively answer the reasons for poor growth throughout the period of childhood. Strategies addressing the nutritional status of women are needed in addition to the assessing the Childhood undernutrition and underweight prevalence in Gamo Gofa zone and child healthcare programs.

\section{Acknowledgement}

The authors would like to thank Arba Minch University Research Directorate Office, College of Natural Sciences Research coordination Offices and Department of Statistics for funding this research and logistic support. Our earnest thanks also go to study participants, data collector and supervisors who spent their valuable time responding to the questionnaires accordingly. 


\section{References}

[1] Besag, J., York, Y. and Mollie, A. (1991). Bayesian Image Restoration with two Applications

[2] Brezger, A. and Lang S. (2006). Generalized Structured Additive Regression based on Bayesian P-Splines. Computational Statistics and Data Analysis, Vol 50,P. 967-991.

[3] CSA (2011).Ethiopian Demographic and Health Survey. Addis Ababa.

[4] Fahrmeir, L. and Lang, S. (2001). Bayesian Inference for Generalized Additive Mixed Models Based on Markov Random Field Priors. Applied Statistics (JRSS C), Vol 50, P. $201-220$.

[5] Fahrmeir, L. and Lang, S. (2001a). Bayesian Inference for Generalized Additive MixedModels Based on Markov Random Field Priors. Applied Statistics (JRSS C), 50, 201220 .

[6] Fahrmeir, L. and Lang, S. (2004). Bayesian Semiparametric Regression Analysis of Multicategorical Time-Space Data.To appear in Ann. Inst. Statist.Math.in Spatial Statistics (with discussion). Ann. Inst. Statist. Math., 43, 1-59.
[7] Kammann EE, Wand MP. Geoadditive Models.Journal of the Royal Statistical Society C. 2003; 52: 1-18.

[8] Khaled, K. (2010). Child Malnutrition in Egypt Using Geoadditive Gaussian and Latent Variable Models.

[9] Lang, S. and Brezger, A. (2001).Bayesian P-Splines. SFB 386 Discussion Paper No. 236. University of Munich.

[10] Mila, A.L.; Yang, X.B. and Carriquiry, A.L. (2003).Bayesian Logistic Regression of Clinical Epidemiology for Uncertainty in Parameter Estimation. Basic Science for Clinical Medicine: Little, Brown and Company, Boston.

\section{Biography}

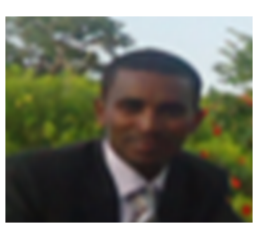

Tilahun Ferede Asena (PhD Candidate)

Mr. Tilahun Ferede Asena: The author of this article has obtained his First Degree (B.Sc) and M.Sc Degree in Applied Statistics in July, 2009 and July, 2012 from Hawassa University respectively. He taught various statistics courses in the previous six years at Arba Minch University, Arba Minch, Ethiopia and he published this article while he was a PhD Student at Hawassa University. 